\title{
Analysis of genitourinary trauma patients admitted to the emergency department
}

\author{
İrfan Aydın, Erdal Yavuz* \\ Department of Emergency Medicine, Adıyaman University, Adıyaman, Turkey
}

\begin{abstract}
Background and objective: Genitourinary injuries are commonly encountered in the emergency department but may be over looked in case of multi-trauma. Determining the clinical features of genitourinary injuries will help physicians in the management of genitourinary trauma.

Methods: The study was conducted in a tertiary hospital. Patients of all ages, admitted in the emergency department, with trauma between 2015 and 2020 were included and analyzed. The cause of genitourinary trauma, affected organs, any accompanying injury, treatments, mortality status, and laboratory tests related to mortality were obtained from the hospital records and analyzed.

Results: During the study period, 87 patients admitted to the emergency department with genitourinary trauma were included in the study. The majority of these patients $(n=79)$ were male. Of the patients, 9.2\% died. All the patients in the mortality group had additional injuries. The most frequently injured organ was determined as the kidney (51.7\%), followed by the scrotum (25.3\%) and penis (8.1\%). Additional injuries were observed in $81.6 \%$ of the patients. Intra-abdominal organ injuries (19.5\%) were the most common accompanying injuries. White blood cell count (WBC), aspartate aminotransferase (AST), alanine aminotransferase ( ALT), blood glucose and creatinine values measured at the time of admission to the emergency department were found to be higher in the non-survivor group. The majority of the patients (81\%) were discharged with conservative treatment and follow-up.
\end{abstract}

Conclusion: It was determined that genitourinary injuries were frequently seen with additional injuries. Genitourinary injury should be evaluated carefully, especially in the presence of intra-abdominal organ injuries.

IMC J Med Sci 2021; 15(2): 005

\section{Introduction}

Traumas constitute a general public health problem and an important cause of mortality and morbidity. Genitourinary injuries occur in approximately 10$20 \%$ of multi-trauma cases and are more common in young men. They usually occur with other lifethreatening injuries that require immediate intervention [1]. The most common causes of genitourinary injuries are traffic accidents, falls, sexual assaults, gunshot wounds, and penetrating stab wounds. Pelvic fractures and abdominal organ damage are the most common injuries accompanied by genitourinary injuries. The most frequently injured organ in genitourinary injuries is the kidney [2].

Evaluation of genital organ damage in trauma patients is performed in a secondary examination. Genitourinary injuries can sometimes be asymptomatic or of secondary importance in case of multi-trauma. Although the mortality rate is low in genitourinary trauma, they are important in terms of their possibility to cause sexual dysfunction and permanent kidney damage. Early diagnosis and treatment of genitourinary injuries are important in preventing or minimizing complications, such as mortality and renal

*Correspondence: Erdal Yavuz, MD, Department of Emergency Medicine, Adıyaman University, 02200, Adıyaman, Turkey.Email: erdal_yavuz15@hotmail.com 
dysfunction, urinary incontinence and sexual dysfunction [3]. As in all trauma cases, in patients with genitourinary injuries, the airway should be protected first, and then external bleeding and hemodynamic shock should be controlled. After evaluating possible causes of fatal trauma, a genitourinary evaluation should be performed in the early period [4]. Thereore, the main purpose of this study was to evaluate genitourinary injuries in trauma patients, discuss them in light of the literature, and draw the attention of emergency physicians to these injuries.

\section{Methods}

Study design and study population: The study was initiated after obtaining approval from the Clinical Research Ethics Committee of Adiyaman university (ethics committee number: 2020/07-34). Informed consent was waived due to the retrospective nature of the study.

In this study, patients from all age groups who presented to our emergency department due to trauma between 2015 and 2020 were examined. Patients with injuries caused by traffic accidents, falls, battery, and sports activities were identified from the hospital archive. The epicrisis reports of these patients were examined, and the patients with genitourinary trauma were recorded. The patients' age, gender, cause of trauma, time of trauma, injured organ, accompanying additional injury, conclusion time of consultation, length of hospital stay, clinical outcomes, and the values of laboratory investigations/tests at the time of admission to the emergency department were recorded in prepared forms. The obtained values were analyzed and compared between the nonsurvivor and survivor groups. Patients without genitourinary trauma and those with missing data in the hospital archive were not included in the study.

Statistical Analysis: SPSS software package version 17 was used in the study. The suitability of continuous data to normal distribution was investigated with the Kolmogorov-Smirnov test. Data conforming to normal distribution were analyzed using Student's t-test, and those that were not normally distributed were analyzed with the Mann-Whitney $\mathrm{U}$ test. The chi-square test was used to compare qualitative data. Numerical data conforming to normal distribution were shown as mean \pm standard deviation, and those that were not normally distributed were presented as median (minimum-maximum) values. Categorical variables were expressed as numbers and percentages. $P$ values of $<0.05$ were considered statistically significant.

\section{Results}

During the study period, a total of 87 patients, 79 $(90.8 \%)$ male and $8(9.2 \%)$ female, presented to the emergency department with genitourinary injuries (Table-1). Concerning age, $36.7 \%$ of the patients consisted of young males aged 21-40 years. The injuries most frequently occurred in the time zone between 16:00 and 23:59 (49.4\%), and when the trauma type was considered, it was most frequently related to traffic accidents (48.3\%).

Table-1: Characteristics of the study patients with genitourinary injuries $(N=87)$

\begin{tabular}{l|c|c}
\hline \multicolumn{1}{c|}{ Variable } & $\mathbf{n}$ & (\%) \\
\hline Gender & & \\
$\quad$ Male & 79 & 90.8 \\
Female & 8 & 9.2 \\
\hline Age, years & & \\
$\leq 20$ & 25 & 28.7 \\
$21-40$ & 32 & 36.7 \\
$41-60$ & 20 & 22.9 \\
$\geq 61$ & 10 & 11.4 \\
\hline Time of injury & & \\
08:00-15:59 & 34 & 39.1 \\
16:00-23:59 & 43 & 49.4 \\
24:00-07:59 & 10 & 11.5 \\
\hline Trauma type & & \\
Traffic accident & 42 & 48.3 \\
Fall from a height & 19 & 21.8 \\
Penetratingwound & 11 & 12.6 \\
Battery & 5 & 5.7 \\
Gunshot wound & 7 & 8.1 \\
Blunt abdominal trauma & 2 & 2.3 \\
Trapped in wreckage & 1 & 1.1 \\
\hline
\end{tabular}


The most common injury type (51.7\%) was kidney injury, followed by scrotal injury $(25.3 \%)$ and penile injury (8.1\%). When accompanying injuries were examined, an additional pathology was observed in $81.6 \%$ of the patients. It was determined that intra-abdominal organ injury (19.5\%) was the most common accompanying injury, followed by pelvis fractures (17.2\%), extremity fractures (13.8\%), and thoracic injury $(12.6 \%)$. All the patients in the non-survivor group had additional pathologies. The majority (72.4\%) of urology consultations requested from the emergency department were completed within the first hour. Almost half (49.4\%) of the patient had to stay in hospital for 5 to 20 days. The mortality rate of patients with urogenital trauma was found to be $9.2 \%$ (Table-2).

The values of serum aspartate amino transferase (AST), alanine amino transferase (ALT), creatinine and blood glucose in the patients who died were higher than the values of the patients who survived according to the tests taken at the time of admission to the emergency department. Hemoglobin ( $\mathrm{Hb})$, hematocrit (HTC) and urea levels were found to be lower in patients who died, and all of these results were statistically significant $(p<0.05)$. There was no significant difference between deceased and surviving patients in terms of white blood cells (WBC), platelet (PLT) and creatinine kinase (CK) values $(p>0.05)$ (Table- 3$)$.
Table-2: Pattern of genitourinary injuries, hospital stay and outcome

\begin{tabular}{l|l|l}
\hline \multicolumn{1}{c|}{ Variables } & $\mathbf{n}$ & \multicolumn{1}{c}{$\%$} \\
\hline Injured organ & & \\
Kidney & 45 & 51.7 \\
Scrotum & 22 & 25.3 \\
Penis & 7 & 8.1 \\
Bladder & 5 & 5.7 \\
Hematuria & 4 & 4.6 \\
Adrenal hematoma & 3 & 3.4 \\
Ureter & 1 & 1.1 \\
\hline Accompanying injury & & \\
Abdominal organ injury & 17 & 19.5 \\
Pelvic fracture & 15 & 17.2 \\
Extremity fracture & 12 & 13.8 \\
Thoracic injury & 11 & 12.6 \\
Cranial injury & 9 & 10.3 \\
Vertebral fracture & 7 & 8.1 \\
None & 16 & 18.4 \\
\hline Time to complete consultation & & \\
<1hour & 63 & 72.4 \\
1-4hours & 17 & 19.5 \\
>4hours & 7 & 8 \\
\hline Length of hospital stay & & \\
<5 days & 36 & 41.4 \\
5-20 days & 43 & 49.4 \\
>20 days & 8 & 9.2 \\
\hline Outcome & 79 & 90.8 \\
Discharged & 8 & 9.2 \\
Non-survivor (Expired) & & \\
\hline & & \\
\hline & &
\end{tabular}

Table-3: Comparative values of laboratory investigations of survivor and non-survivors cases with genitourinary injuries

\begin{tabular}{lccc}
\hline Investıgations & $\begin{array}{c}\text { Non-survivor group } \\
\text { (Mean } \pm \text { SD) }\end{array}$ & $\begin{array}{c}\text { Survivor group } \\
\text { (Mean } \pm \text { SD) }\end{array}$ & p value \\
\hline Total WBC $\left(\times 10^{9} / \mathrm{L}\right)$ & $16,86 \pm 10.1$ & $15.04 \pm 5.9$ & 0.877 \\
Hemoglobin $(\mathrm{g} / \mathrm{dL})$ & $10,54 \pm 3.6$ & $14,22 \pm 2.1$ & $\mathbf{0 . 0 0 2}$ \\
Hematocrit $(\%)$ & $32,29 \pm 11.7$ & $42,8 \pm 6.2$ & $\mathbf{0 . 0 1 2}$ \\
Total platelet $\left(\times 10^{9} / \mathrm{L}\right)$ & $238,6 \pm 95.7$ & $253,4 \pm 60.1$ & 0.831 \\
S. Urea $(\mathrm{mg} / \mathrm{dL})$ & $25,88 \pm 6.6$ & $33,66 \pm 10.2$ & $\mathbf{0 . 0 2 6}$ \\
S. Creatinine $(\mathrm{mg} / \mathrm{dL})$ & $1,13 \pm 0.34$ & $0,92 \pm 0.19$ & $\mathbf{0 . 0 4 9}$ \\
S. AST $(\mathrm{U} / \mathrm{L})$ & $426 \pm 726.9$ & $98 \pm 149.6$ & $\mathbf{0 . 0 0 5}$ \\
S. ALT $(\mathrm{U} / \mathrm{L})$ & $255 \pm 301.5$ & $87 \pm 154.6$ & $\mathbf{0 . 0 0 2}$ \\
Blood glucose $(\mathrm{mg} / \mathrm{dL})$ & $233.8 \pm 131.3$ & $132.9 \pm 41.1$ & $\mathbf{0 . 0 0 2}$ \\
S. Creatinine kinase (U/L) & $678 \pm 276.9$ & $891 \pm 685$ & 0.338 \\
\hline
\end{tabular}

Note: WBC - white blood cell; AST - aspartate aminotransferase; ALT-alanine aminotransferase; $S$ - serum. 
Considering the interventions performed on the patients, $58 \%$ of the patients were hospitalized with medical treatment, $23 \%$ were followed up on an outpatient basis, $18 \%$ received surgical treatment, and one underwent arterial embolization (Figure-1).

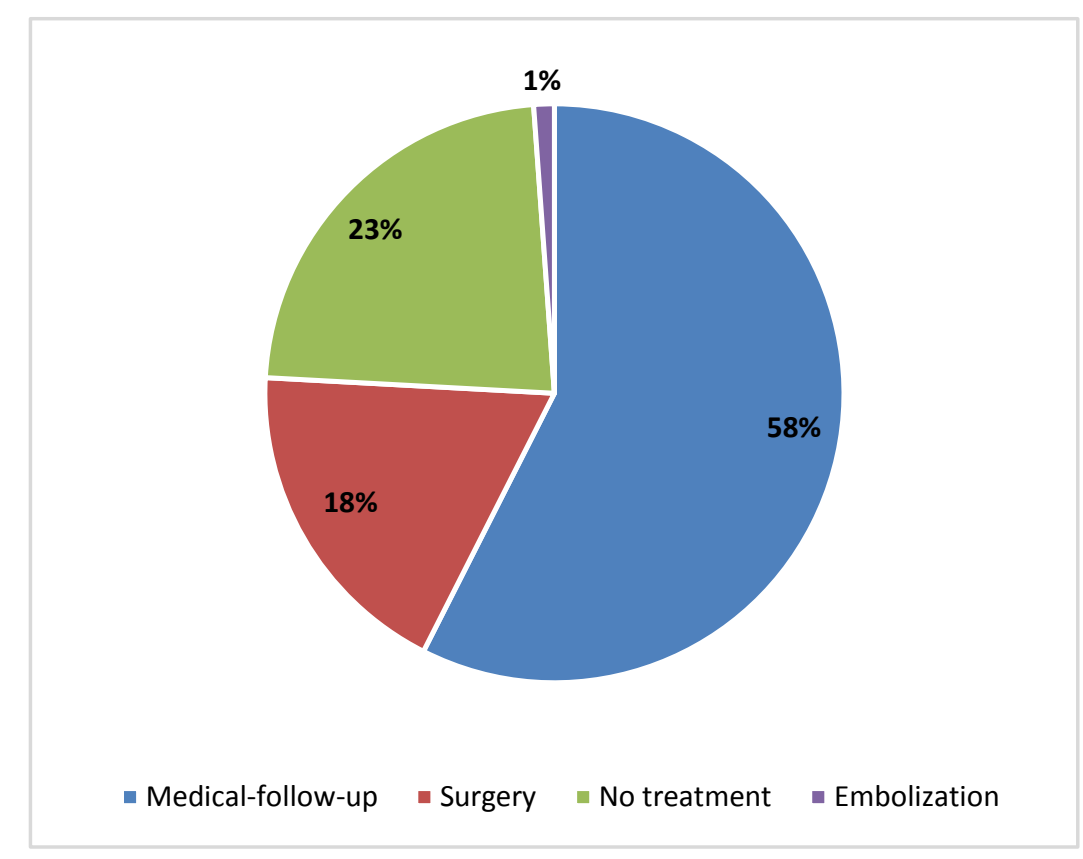

Figure-1: Interventions performed on patients with genitourinary injuries

\section{Discussion}

The first place to which most trauma patients are refered is the emergency department. Genitourinary injuries accompanying multiple traumas may be overlooked during the first examination [5]. Genitourinary injuries can cause significant morbidity and mortality [6]. Although genitourinary injuries can be seen in both genders, their incidence is higher in young men [7]. The majority of the patients in our study consisted of males aged $21-40$ years. This could be due to the greater exposure of young males to trauma. Trauma is the cause of $10 \%$ of all mortality. The mortality rate is lower in isolated genitourinary injuries [8]. In our study, the mortality rate was found to be $9.2 \%$, and additional pathologies were found in all of these patients. None of the patients with isolated genitourinary trauma died. Multiple organ injuries involving more than one system were found to increase the mortality.
Traffic accidents are one of the most important causes of multi-trauma and have an increasing global incidence [9]. Buyukcam et al. [10] reported that the most common cause of genitourinary traumas was traffic accidents at a rate of $52 \%$. Similarly, in our study, traffic accidents constituted the most common cause of genitourinary traumas at a rate of $54 \%$. Although the kidney is a wellprotected organ, it is the most frequently injured organ in genitourinary trauma [11]. The most common injury is due to blunt trauma. Previous studies found that $80-90 \%$ of kidney traumas were accompanied by additional pathologies [12.13]. In the current study, the most frequently injured organ was the kidney $(51.7 \%)$. The rate of pathologies accompanying the genitourinary traumas was $81.4 \%$, which was in agreement with other studies. However, contrary to our study, Bagga et al. [14] found that external genital organ injuries were the most common. We believe that more studies are needed on this subject. 
Recently, the recommended mangement approach in kidney trauma is conservative treatment. Arterial embolization is recommended in patients with active bleeding. Buyukcam et al. [10] reported that only 21 of 153 patients underwent surgery. In our study, 16 of the patients were treated in the operating room. Arterial embolization was applied to only one patient. Genitourinary injuries should be followed up closely in terms of hemodynamics.

In trauma patients, changes in laboratory values can be seen due to coagulopathy and organ damage. Coagulopathy is seen in $25 \%$ of severe traumas [15]. In our study, lower hemoglobin and platelet and higher WBC values were observed among the cases in the non-survivor group, which might be due to bleeding and coagulopathy. In addition, higher AST and ALT levels among the nonsurvivors indicate the presence of accompanying intra-abdominal organ injuries.

\section{Conclusion}

Genitourinary trauma is often seen together with additional pathologies. Genitourinary injuries should be carefully examined, especially when there are intra-abdominal organ injuries. In patients with genitourinary injuries, laboratory values evaluated at the time of admission to the emergency department can provide clues about the possibility of fatal outcome. Further research is needed in this area.

Authorship: EY and IA contributed to conception and design, supervision; IA contributed to data collection and processing; $E Y$ contributed to analysis and interpretation; EY, IA contributed to literature review; EY contributed to writing; and IA contributed to critical review.

Funding: The author(s) received no financial support for the research, authorship, and/or publication of this article.

Conflict of interest: The author(s) declared no potential conflicts of interest with respect to the research, authorship, and/or publication of this article.

Human rights: Authors declare that human rights were respected according to Declaration of Helsinki.

\section{References}

1. Gauss T, Ageron FX, Devaud ML, Debaty G, Travers S, Garrigue D et al. Association of prehospital time to in-hospital trauma mortality in a physician-staffed emergency medicine system. JAMA Surg. 2019; 154(12): 1117-1124.

2. Kaya C, Koca O, Kalkan S, Oztürk M, İlktaç A, Karaman IM. [Evaluation of patients with urogenital trauma managed in a urology clinic]. Ulus Travma Acil Cerrahi Derg. 2009; 15(1): 67-70. Turkish

3. Cutinha P, Venugopal S, Salim F. Genitourinary trauma. Surgery (Oxford). 2013; 31(7): 362-370.

4. Wardak SW, Nuttall MC. Genitourinary trauma. Surgery (Oxford). 2016; 34(7): 361-368.

5. Smith, CM, Woolrich-Burt L, Wellings R, Costa, ML. Major trauma CT scanning: the experience of a regional trauma centre in the UK. Emerg Med J. 2011; 28(5): 378-382.

6. Bryk DJ, Zhao LC. Guideline of guidelines: a review of urological trauma guidelines. BJU Int. 2016; 117(2): 226-234.

7. Djakovic N, Plas E, Martínez-Piñeiro L, Lynch TH, Mor $Y$ et al. Guidelines on urological trauma. EAU Guidelines. 2009; 31-35.

8. Mc Geady JB, Breyer BN. Current epidemiology of genitourinary trauma. Urol clin North Am. 2013; 40(3): 323.

9. Ramadani N, Zhjeqi V, Berisha M, Hoxha R, Begolli I, Salihu D et al. Public health profile of road traffic accidents in Kosovo 20102015. Open access Maced J Med Sci. 2017; 5(7): 1036.

10. Büyükcam F, Şen J, Akpınar Ş, Zengin $Y$, Çalık $M$, Odabaş Ö. [Evaluation of urogenital injuries in patients with trauma in the Emergency Department]. Ulus Travma Acil Cerrahi Derg. 2012; 18(2): 133-140. Turkish

11. Phillips B, Holzmer S, Turco L, Mirzaie $M$ Mause E, Mause A et al. Trauma to the bladder and ureter: a review of diagnosis, management, and prognosis. Eur J Trauma Emerg Surg. 2017; 43(6); 763-773. 
12. Van der Wilden GM, Velmahos GC, Joseph DK. Successful non operative management of the most severe blunt renal injuries: a multicenter study of the research consortium of New England Centers for Trauma. JAMA Surg. 2013; 148: 924-931.

13. Kansas BT, Eddy MJ, Mydlo JH. Incidence and management of penetrating renal trauma in patients with multiorgan injury: extended experience at an inner city trauma center. J Urol. 2004; 172: 1355-1360.
14. Bagga, HS, Tasian GE, Fisher PB, McCulloch $\mathrm{CE}$, McAninch JW, Breyer BN. Product related adult genitourinary injuries treated at emergency departments in the United States from 2002 to 2010. J Urol. 2013; 189(4): 1362-1368.

15. Erenler AK, Oğuzhan AY M. [Coagülopaty in patients with trauma]. Turkiye Klinikleri J Emerg Med-Special Topics. 2017; 3(1): $103-$ 107. Turkish. 